\title{
GHB dans le sang post-mortem. Critères d'interprétation
}

\section{Interpretation of postmortem $G H B$ concentrations}

\section{Pascal KINTZ*, Marion VILLAIN, Carole JAMEY, Vincent CIRIMELE, Antoine TRACQUI, Bertrand LUDES}

Institut de Médecine Légale, 11, rue Humann - F-67000 Strasbourg - France

*Auteur à qui adresser la correspondance : Dr Pascal KINTZ, Institut de Médecine Légale, 11, rue Humann - F-67000 Strasbourg - France Tél : +33 390243349 - Fax : +33 390243362 - E-mail : pascal.kintz@wanadoo.fr

(Reçu le 8 mars 2004 ; accepté le 15 juin 2004)

\section{$R \hat{E} S U M \dot{E}$}

Il est désormais admis par la communauté scientifique que $d u$ GHB peut être retrouvé dans le sang post-mortem en absence de toute exposition à ce stupéfiant. Dans ces conditions, un seuil analytique de positivité a été proposé à 50 mg/l dans le sang. Afin de valider ce seuil, 73 échantillons de sang cardiaque post-mortem, obtenus lors d'autopsies où le médecin légiste a pu exclure tout lien avec le GHB, ont été analysés par GC/MS après précipitation. Le délai entre le décès et l'autopsie a varié entre 12 et 72 heures. En parallèle, lorsque cela était possible, du sang périphérique, de la bile et de l'humeur vitrée ont été également analysés.

Il a été retrouvé $d u$ GHB dans tous les échantillons de sang cardiaque, avec des concentrations variant de 0,4 à 409 $m g / l$.

Une concentration supérieure à $50 \mathrm{mg} / \mathrm{l}$ a été observée dans 16 cas. Les examens complémentaires, avec les autres prélèvements correspondants, ont donné les résultats suivants : - sang cardiaque (51 à $409 \mathrm{mg} / \mathrm{l}$ ) versus sang périphérique (1,6 ̀̀ $44 \mathrm{mg} / \mathrm{l})$ dans $6 \mathrm{cas}$

- sang cardiaque (55 à $409 \mathrm{mg} / \mathrm{l}$ ) versus bile (6 à $238 \mathrm{mg} / \mathrm{l}$ )

\section{SUMMARY}

Since GHB is present in both blood and urine of the general population as an endogenous compound, toxicologists must be able to discriminate between these endogenous levels and a concentration resulting from exogenous exposure.

To verify the accuracy of a proposed $50 \mathrm{mg} / \mathrm{L}$ postmortem blood cut-off, we tested 73 autopsy cases of subjects where the cause of death could exclude GHB exposure. The delay between death and autopsy ranged from 12 to 72 hours. $G H B$ was tested by GC/MS after precipitation. Briefly, $20 \mu L$ blood, bile, or vitreous humor were pipetted in a glass tube,

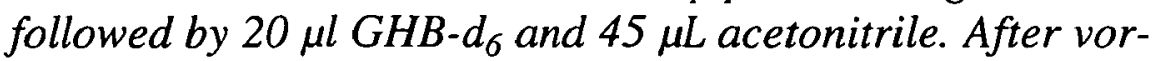
texing and centrifugation, the supernatant was collected and evaporated to dryness. The residue was derivatized with BSTFA with $1 \%$ TMCS for $20 \mathrm{~min}$ at $70^{\circ} \mathrm{C}$. GHB $(\mathrm{m} / \mathrm{z} 233$, 204 and 147) and $G H B-d_{6}(m / z 239)$ were identified by $M S$. GHB tested positive in all the 73 whole blood (cardiac) specimens, with concentrations in the range 0.4 to $409 \mathrm{mg} / \mathrm{l}$, with a major distribution in the range $10-40 \mathrm{mg} / \mathrm{l}$. A concentration $>50 \mathrm{mg} / \mathrm{l}$ was observed in 16 cases. As there was no data to support GHB exposure, this was considered as post- 
dans 9 cas

- sang cardiaque (51 à $409 \mathrm{mg} / \mathrm{l}$ ) versus humeur vitrée (4 à $21 \mathrm{mg} / \mathrm{l})$ dans 7 cas

Il apparaît que l'application stricte d'un seuil de positivité dans le sang à $50 \mathrm{mg} / \mathrm{l}$ ne permet pas d'interpréter correctement les données analytiques. La bile ne semble pas d'un intérêt discriminant et seuls le sang périphérique, mais surtout l'humeur vitrée permettent de documenter au mieux les résultats.

Il est essentiel de confirmer toute concentration de GHB supérieure à $50 \mathrm{mg} / \mathrm{l}$ dans le sang cardiaque par une détermination concomitante dans le sang périphérique et l'humeur vitrée. L'urine pouvant également contenir des concentrations importantes de GHB, n'apporte pas d'information complémentaire. Des travaux sont en cours pour confirmer l'intérêt de la détermination du rapport isotopique ${ }^{12} \mathrm{C}^{3} \mathrm{C}$ (comme pour la testostérone) pour différencier un usage exogène d'une formation endogène.

\section{MOTS-CLÉS}

GHB, post-mortem, sang, humeur vitrée, interprétation.

\section{Introduction}

Le GHB est un produit naturellement présent chez l'homme à des concentrations nanomolaires.

Son sel de sodium, classé comme stupéfiant depuis le 5 mai 1999 est une poudre blanche, micro-cristalline, fortement hygroscopique, soluble dans l'eau et de saveur salée. On le rencontre sur le marché noir sous forme de liquide translucide, pouvant être parfois coloré, dont les concentrations sont très variables.

L'administration de GHB entraîne une importante augmentation des concentrations en dopamine au niveau central. Il protège le SNC des effets délétères d'une demande métabolique excessive ou d'une hypoxie, ce qui en fait son intérêt en anesthésiologie.

Son usage thérapeutique est ancien, l'AMM initial datant de 1961. Celle-ci a été renouvelée le 14 janvier 1998, avec comme indications thérapeutiques la sédation en neuro-traumatologie et la propriété d'adjuvant anesthésique en chirurgie et obstétrique.

Sur le plan pharmacologique, le GHB fait partie de la classe des hypnotiques et anesthésiques, avec des indications comme inducteur de sommeil ou comme adjuvant des analgésiques et autres anesthésiques en chirurgie. La posologie usuelle est de $60 \mathrm{mg} / \mathrm{kg}$ chez l'adulte et $100 \mathrm{mg} / \mathrm{kg}$ chez l'enfant. Il produit hypnose et hypotonie, mais n'a pas d'effet analgésique ou myorelaxant, les myoclonies ou les convulsions sont donc possibles. L'administration IV provoque une augmentation modérée et transitoire de la tension artérielle, associée à une bradycardie et à une diminution de la fréquence respiratoire. Le GHB est également un hypothermisant, un hypokaliémiant et un ocytocique (1). mortem formation. In order to discriminate this contamination, when available, femoral blood, bile orland vitreous humor were tested. The following results were obtained : - cardiac bood (55 to $409 \mathrm{mg} / \mathrm{l}$ ) versus bile (6 to $238 \mathrm{mg} / \mathrm{l}$ ) in 9 cases ;

- cardiac blood (51 to $409 \mathrm{mg} / \mathrm{l}$ ) versus femoral blood (2 to 44) in 6 cases, and

- cardiac blood (51 to $409 \mathrm{mg} / \mathrm{l}$ ) versus vitreous humor (4 to 21) in 7 cases.

It is obvious that bile does not fit the requirements for discrimination and that femoral blood and mostly vitreous humor can be of particular interest. These results demonstrate that a positive (> $50 \mathrm{mg} / \mathrm{l}$ ) postmortem blood $\mathrm{GHB}$ concentration cannot support alone drug exposure and that it is essential to document the case with other specimens, including peripheral blood and vitreous humor.

\section{KEY-WORDS}

$G H B$, postmortem, blood, vitreous humor, interpretation.

La demi-vie du GHB est proportionnelle à la dose administrée, probablement due à une limitation de sa dégradation enzymatique. Par voie IV, elle est de 40 min pour une dose à $60 \mathrm{mg} / \mathrm{kg}$, per os elle est de 20 à $25 \mathrm{~min}$ pour des doses de 12,5 à $50 \mathrm{mg} / \mathrm{kg}$, avec un pic plasmatique atteint en 20 à $45 \min (2)$.

Dès 1998, et la banalisation de l'usage du GHB aux Etats-Unis dans les affaires de soumission chimique ou de conduite automobile sous influence, la presse scientifique mettait en garde les toxicologues sur une possible formation in vitro (3). Ces auteurs observaient dans 15 autopsies sur 20 des concentrations sanguines de GHB de 3,2 à $168 \mathrm{mg} / \mathrm{l}$ (moyenne à $25 \mathrm{mg} / \mathrm{l}$ ), alors que les sujets n'étaient pas à priori exposés au GHB. Dans tous les cas, les urines étaient restées négatives. Ces résultats étaient confirmés par d'autres travaux, dont par exemple ceux de Stephens et coll. (4). L'interprétation se compliquait en 2001, avec une publication (5) mentionnant une formation post-mortem également dans les urines. En 2002, Kintz (6) proposait un seuil de positivité à $50 \mathrm{mg} / \mathrm{l}$ dans le sang postmortem, associé à la détection du GHB dans les urines.

Afin de documenter la présence de GHB dans le sang post-mortem et son interprétation, nous avons analysé ce produit dans 73 expertises médico-légales consécutives, dont les prélèvements ont été effectués à l'IML de Strasbourg. 


\section{Matériel et méthodes Échantillons biologiques}

Du sang cardiaque, du sang fémoral, de la bile et de l'humeur vitrée ont été recueillis lors d'autopsies sur réquisition du Parquet de Strasbourg. Compte tenu des données de la littérature, les urines ont été considérées comme sans intérêt. Aucun conservateur n'a été ajouté aux échantillons. Le délai entre le décès et l'autopsie était généralement compris entre 12 et 72 heures. Dans tous les 73 cas, la cause de la mort n'avait aucun lien avec le GHB, comme ont pu l'estimer les médecins légistes. Avant analyse, faite sous 48 heures, les échantillons ont été conservés à $+4^{\circ} \mathrm{C}$.

Le GHB a été analysé dans tous les 73 échantillons de sang cardiaque. Lorsque la concentration excédait $50 \mathrm{mg} / \mathrm{l}$, le GHB a été dosé dans les autres échantillons biologiques, lorsqu'ils étaient disponibles.

\section{Réactifs}

L'acétonitrile est de qualité HPLC (Merck, Darmstadt, Allemagne). Le BSTFA + $1 \%$ TMCS (N,O-bis(triméthylsilyl)trifluoroacétamide $+1 \%$ triméthylchlorosilane) est acheté chez Fluka (Saint-Quentin Fallavier, France). Le GHB et le GHB-d $\mathrm{d}_{6}$ proviennent de Promochem (Molsheim, France).

\section{Analyse du GHB}

La procédure est identique, quel que soit le fluide biologique analysé et découle des travaux de Villain et al (7).

Brièvement, à $20 \mu \mathrm{l}$ de sang total, de bile ou d'humeur vitrée, on ajoute $5 \mu \mathrm{l}$ de GHB- $\mathrm{d}_{6}$ (standard interne) à $100 \mathrm{mg} / \mathrm{l}$ et $45 \mu \mathrm{l}$ d'acétonitrile pour précipiter les protéines. Après agitation au vortex et centrifugation, la phase surnageante est prélevée puis évaporée à sec. Le résidu est repris par $35 \mu \mathrm{l}$ de BSTFA + $1 \%$ TMCS pour former des dérivés silylés qui sont injectés sur une colonne HP5-MS (5\% phenyl-95 \% méthylsiloxane, $30 \mathrm{~m} \times 0.25 \mathrm{~mm}$ i.d. $\times 0.25 \mathrm{~m}$ ) d'un système de GC/MS. Le GHB est identifié par ses ions $(\mathrm{m} / \mathrm{z} 233$, 204 et 159$)$ et quantifié par rapport au GHB-d ${ }_{6}(\mathrm{~m} / \mathrm{z}$ 239). La limite de détection de la méthode est de l'ordre de $0,5 \mathrm{mg} / \mathrm{l}$, largement suffisante pour les besoins toxicologiques.

Les dérivés sont stables au moins 24 heures.

\section{Résultats}

Dans un cas de décès, Marinetti et coll. (8) ont retrouvé du GHB dans le sang cardiaque à la concentration de $66 \mathrm{mg} / \mathrm{l}$. Après une exposition unique et létale, Kalasinsky et coll. (9) ont dosé le GHB à $330 \mathrm{mg} / \mathrm{l}$ dans le sang fémoral. Une concentration post-mortem de $538 \mathrm{mg} / \mathrm{l}$ de GHB a été mise en évidence après ingestion de gamma-hydroxybutyrolactone (10). De ces concentrations post-mortem, il apparaît que l'intervalle des valeurs potentiellement létales est important, probablement lié à la rapide élimination du produit du compartiment sanguin.

Les concentrations de GHB dans le sang cardiaque sont données dans le Tableau I. La distribution de ces concentrations est représentée Fig 1. Dans tous les cas, une exposition au GHB a pu être exclue par les médecins légistes expérimentés et les données de la Police. Le GHB a été retrouvé dans tous les 73 échantillons de sang cardiaque avec des concentrations comprises entre 0,4 et $409 \mathrm{mg} / \mathrm{l}$, et une distribution majeure entre 10 et $40 \mathrm{mg} / 1$. Tout comme l'avait observé Elliott (11), il n'y a pas de corrélation apparente entre la concentration sanguine en GHB et la cause du décès.

Une concentration supérieure à $50 \mathrm{mg} / \mathrm{l}$ (seuil de positivité usuel) a été observée dans 16 cas ( 51 à 409 mg/l). Lorsqu'ils étaient disponibles, le sang fémoral, la bile et l'humeur vitrée ont alors été analysés. Les résultats des analyses sont donnés dans les Tableaux II, III et IV.

Marinetti et coll. (8) ont retrouvé, dans un cas d'intoxication mortelle, du GHB dans le sang cardiaque $(66 \mathrm{mg} / \mathrm{l})$, fémoral $(77 \mathrm{mg} / \mathrm{l})$ et l'humeur vitrée (85 $\mathrm{mg} / \mathrm{l})$. Dans un cas de décès d'un héroïnomane ayant également abusé du GHB (12), les concentrations suivantes étaient retrouvées : sang $(12 \mathrm{mg} / \mathrm{l})$, bile $(57$ $\mathrm{mg} / \mathrm{l})$ et humeur vitré $(84 \mathrm{mg} / \mathrm{l})$. Il semble donc que l'humeur vitrée soit un milieu d'accumulation en cas d'exposition au GHB.

Au contraire, dans notre cohorte de sujets non-exposés au GHB, même en cas de concentrations élevées dans le sang cardiaque, les concentrations dans le sang fémoral (2 à $44 \mathrm{mg} / \mathrm{l})$ et l'humeur vitrée $(4$ à $21 \mathrm{mg} / \mathrm{l})$ sont restées modestes.

C'est pourquoi, les auteurs recommandent, afin de pouvoir attribuer au GHB une responsabilité dans le processus létal, de faire simultanément le dosage dans le sang fémoral et l'humeur vitrée, en utilisant un seuil de positivité à $50 \mathrm{mg} / \mathrm{l}$. Par contre, le dosage dans la bile (6 à $238 \mathrm{mg} / \mathrm{l})$ n'apporte pas de possibilité de disćrimination.

Une étude récente (13) a montré qu'il n'y avait pas de corrélation entre la concentration sanguine de GHB et la concentration des marqueurs de putréfaction comme la tryptamine ou la phényl-2-éthylamine, les auteurs concluant à une production microbienne du GHB dans le temps.

Selon notre expérience, la formation post-mortem du GHB serait fonction du délai entre le décès et le 
Tableau I : Concentrations de GHB dans le sang cardiaque et cause de la mort dans 73 cas.

\begin{tabular}{|c|c|c|}
\hline Cas & GIBB (img/1) & Cause de la mort \\
\hline 1 & 0,9 & Viol + homicide \\
\hline $2 *$ & 223,6 & Intoxication au $\mathrm{CO}$ \\
\hline 3 & 1,0 & Décès naturel \\
\hline 4 & 11,6 & Suicide par arme à feu \\
\hline 5 & 0,4 & Homicide \\
\hline 6 & 7,0 & Décès naturel \\
\hline $7 *$ & 95,0 & Noyade \\
\hline 8 & 28,6 & Pendaison \\
\hline 9 & 3,7 & Suicide \\
\hline 10 & 48,4 & Homicide \\
\hline 11 & 44,1 & Intoxication médicamenteuse \\
\hline 12 & 17,9 & Intoxication médicamenteuse \\
\hline 13 & 30,0 & Asphyxie \\
\hline 14 & 10,5 & Noyade \\
\hline 15 & 11,6 & Décès naturel \\
\hline 16 & 22,1 & Décès naturel \\
\hline 17 & 29,7 & Intoxication médicamenteuse \\
\hline 18 & 27,8 & Noyade \\
\hline 19 & 7,7 & Asphyxie \\
\hline 20 & 22,2 & Tué par un train \\
\hline 21 & 4,1 & Décès naturel \\
\hline 22 & 0,7 & Décès naturel \\
\hline 23 & 12,8 & Homicide \\
\hline 24 & 3,2 & Intoxication au $\mathrm{CO}$ \\
\hline 25 & 8,0 & Homicide \\
\hline $26^{*}$ & 409 & Alcoolisme chronique \\
\hline 27 & 14,2 & Décès naturel \\
\hline 28 & 6,7 & Overdose par cocaïne \\
\hline 29 & 7,2 & Overdose par cocaine \\
\hline 30 & 11,9 & Asphyxie \\
\hline $31 *$ & 55,5 & Accident de la voie publique \\
\hline 32 & 34,5 & Décès naturel \\
\hline $33^{*}$ & 85,4 & Intoxication par carbamazépine \\
\hline 34 & 42,9 & Saut dans le vide \\
\hline 35 & 2,0 & Noyade \\
\hline 36 & 1,2 & Homicide \\
\hline 37 & 1,0 & Overdose par héroïne \\
\hline $38^{*}$ & 55,3 & Homicide \\
\hline $39 *$ & 75,4 & Chute \\
\hline 40 & 17,8 & Homicide \\
\hline 41 & 13,5 & Incendie \\
\hline $42 *$ & 67,5 & Epilepsie \\
\hline $43^{*}$ & 89,6 & Noyade \\
\hline 44 & 5,8 & Décès naturel \\
\hline 45 & 3,8 & Homicide \\
\hline 46 & 38,4 & Décès naturel \\
\hline 47 & 13,9 & Noyade \\
\hline 48 & 24,1 & Accident de la voie publique \\
\hline 49 & 31,6 & Pendaison \\
\hline 50 & 4,9 & Décès naturel \\
\hline 51 & 48,5 & Décès naturel \\
\hline 52 & 6,5 & Décès naturel \\
\hline $53 *$ & 54,6 & Intoxication médicamenteuse \\
\hline $54^{*}$ & 93,6 & Intoxication médicamenteuse \\
\hline 55 & 15,2 & Overdose par héroïne \\
\hline $56^{*}$ & 50,8 & Décès naturel \\
\hline 57 & 18,5 & Homicide \\
\hline 58 & 5,9 & Accident de la voie publique \\
\hline 59 & 10,5 & Accident de la voie publique \\
\hline 60 & 10,2 & Intoxication médicamenteuse \\
\hline 61 & 9,6 & Overdose par héroïne \\
\hline 62 & 135 & Noyade \\
\hline $63^{*}$ & 262 & Noyade \\
\hline 64 & 16,7 & Homicide \\
\hline 65 & 19,3 & Décès naturel \\
\hline 66 & 46,9 & Intoxication par méthadone \\
\hline 67 & 9,8 & Overdose par héroïne \\
\hline 68* & 56,3 & Incendie \\
\hline 69 & 4,4 & Noyade \\
\hline 70 & 22,3 & Accident de la voie publique \\
\hline 71 & 42,6 & Intoxication médicamenteuse \\
\hline $72 *$ & 173,9 & Overdose par héroïne \\
\hline $73^{*}$ & 149,8 & Décès naturel \\
\hline
\end{tabular}

moment du recueil de l'échantillon sanguin (moment de la levée de corps ou de l'autopsie) et non pas du délai entre le moment du prélèvement et l'analyse, comme le démontrent les résultats du Tableau V. En effet, dans un cas d'accident de la voie publique, le corps d'un homme de 37 ans a été déposé à la morgue de l'MML, dans les 2 heures qui ont suivi son décès. Nous avons prélevé $0,5 \mathrm{ml}$ de sang périphérique à des temps variables pendant 72 heures, directement sur le cadavre et la concentration de $\mathrm{GHB}$ a régulièrement augmenté dans le temps. Au contraire, 2 échantillons prélevés et congelés immédiatement, puis analysés 2 et 8 semaines plus tard n'ont pas montré d'élévation significative des concentrations.

Enfin, des investigations complémentaires sont en cours pour évaluer l'intérêt du rapport isotopique du carbone ${ }^{12} \mathrm{C} /{ }^{13} \mathrm{C}$, tout comme cela est utilisé dans. le dopage pour discriminer un usage exogène de testostérone. Les premiers résultats semblent indiquer un $\partial{ }^{13} \mathrm{C}$ de l'ordre de $-50 \%$ à $-40 \%$ pour le GHB retrouvé dans la rue (comme stupéfiant), qui devrait être suffisant face à un $\partial{ }^{13} \mathrm{C}$ attendu d'environ $-20 \%$ pour le GHB endogène.

\section{Conclusion}

Ces résultats démontrent que la mesure isolée d'une concentration sanguine post-mortem de GHB supérieure à $50 \mathrm{mg} / \mathrm{l}$ ne permet pas à elle seule de conclıre à une exposition. Il convient de doser simultanément le GHB dans le sang fémoral et l'humeur vitrée, tout en conservant pour ces prélèvements un seuil de positivité à $50 \mathrm{mg} / \mathrm{l}$. Il semble que le délai entre le décès et le moment du recueil du prélèvement soit déterminant. Il convient donc que cette période soit la plus courte possible. 
Annales de Toxicologie Analytique, vol. XVI, $n^{\circ} 2,2004$

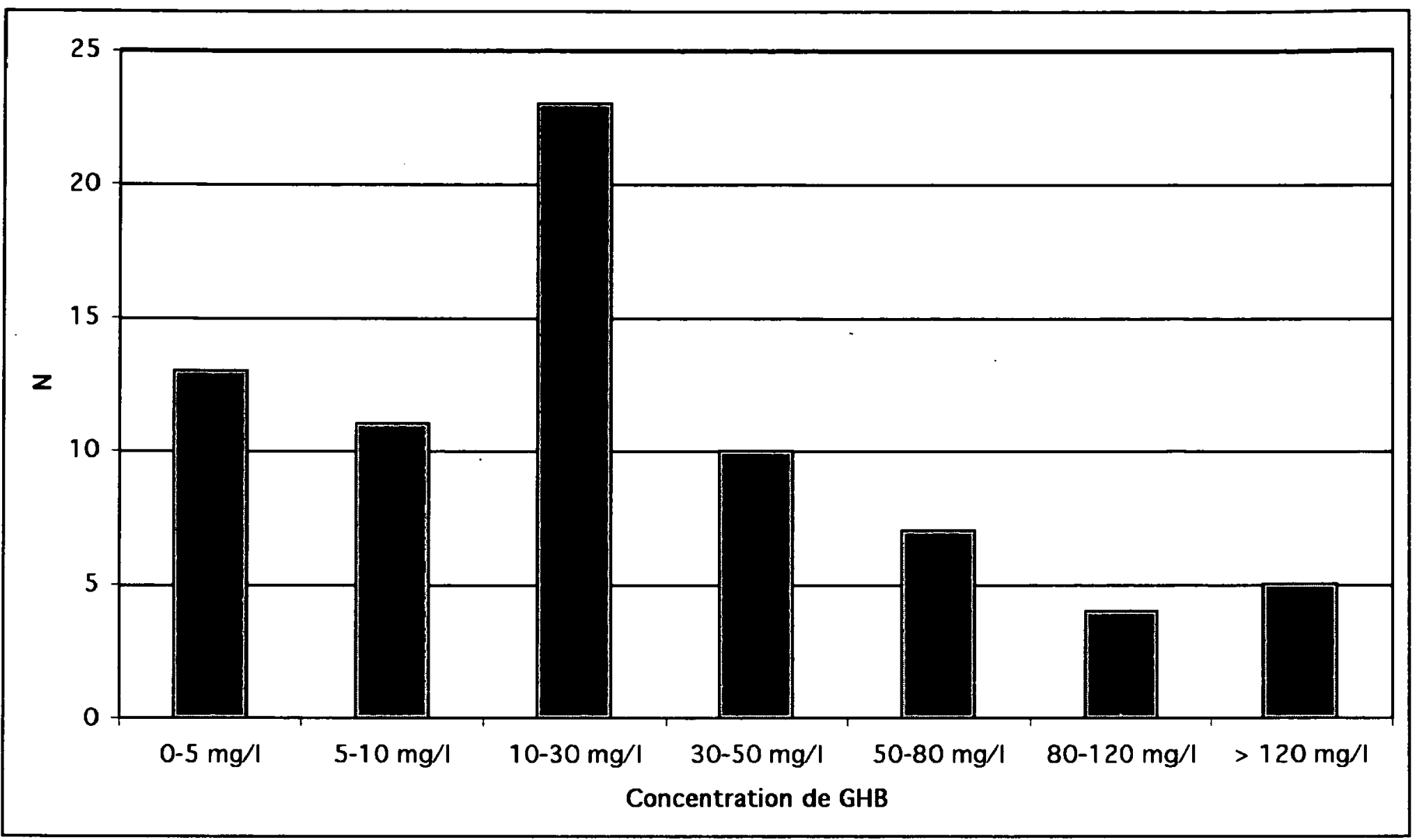

Figure 1 : Distribution du GHB dans le sang cardiaque.

Tableau II : Concordances des concentrations de GHB dans le sang cardiaque et la sang fémoral.

\begin{tabular}{|c|c|c|}
\hline Cas & Sang cardiaque (mg/) & Sang fémoral (mg/) \\
\hline 1 & 409 & 16,8 \\
\hline 2 & 54,6 & 44,1 \\
\hline 3 & 96,6 & 17,1 \\
\hline 4 & 50,8 & 39,7 \\
\hline 5 & 56,3 & 34,3 \\
\hline 6 & 149,8 & 1,6 \\
\hline Moyenne & 136,2 & 25,6 \\
\hline
\end{tabular}

Tableau IV : Concordances des concentrations de GHB dans le sang cardiaque et l'humeur vitrée.

\begin{tabular}{|c|c|c|}
\hline Cas & Sang cardiaque (mg/) & Humeur vitrée (mg/) \\
\hline 1 & 409,0 & 3,9 \\
\hline 2 & 54,6 & 11,6 \\
\hline 3 & 55,5 & 11,5 \\
\hline 4 & 50,8 & 10,8 \\
\hline 5 & 75,4 & 21,4 \\
\hline 6 & 93,6 & 14,5 \\
\hline 7 & 149,8 & 5,1 \\
\hline Moyenne & 126,8 & 11,3 \\
\hline
\end{tabular}

Tableau III : Concordances des concentrations de GHB dans le sang cardiaque et la bile.

\begin{tabular}{|c|c|c|}
\hline Cas & Sang cardiaque (mg/l) & Bile (mg/l) \\
\hline 1 & 409,0 & 238 \\
\hline 2 & 223,6 & 35,5 \\
\hline 3 & 95,0 & 104,9 \\
\hline 4 & 85,4 & 43,3 \\
\hline 5 & 55,3 & 6,1 \\
\hline 6 & 93,6 & 25,7 \\
\hline 7 & 262,0 & 152,0 \\
\hline 8 & 173,9 & 12,0 \\
\hline 9 & 149,8 & 10,8 \\
\hline Moyenne & 172,0 & 69,8 \\
\hline
\end{tabular}

Tableau $V$ : Évolution des concentrations dans le sang de GHB en fonction du temps.

\begin{tabular}{|c|c|}
\hline Moment du prélèvement & Concentration en GHB \\
\hline $\mathrm{T}+3$ heures & $3,5 \mathrm{mg} / 1$ \\
\hline $\mathrm{T}+24$ heures & $9,2 \mathrm{mg} / 1$ \\
\hline $\mathrm{T}+48$ heures & $17,8 \mathrm{mg} / 1$ \\
\hline $\mathrm{T}+72$ heures & $22,9 \mathrm{mg} / 1$ \\
\hline Congélation* $\mathrm{T}+2$ semaines & $3,8 \mathrm{mg} / \mathrm{l}$ \\
\hline Congélation* $\mathrm{T}+8$ semaines & $4,1 \mathrm{mg} / 1$ \\
\hline
\end{tabular}

Cas d'un homme de 37 ans, décédé lors d'un accident de la voie publique et dont le corps a été déposé à l'IML 2 heures après son décès $(\mathrm{T}+2$ heures). Prélèvement in corpore de sang périphérique à des temps variables.

* congélation à $\mathrm{T}+3$ heures de 2 aliquotes pour analyse ultérieure à $\mathrm{T}+2$ semaines et à $\mathrm{T}+8$ semaines. 


\section{Références}

1. Deveaux M. L'acide $\gamma$-hydroxybutyrique (GHB) : un vieux produit, de nouveaux problèmes. J. Anal. Toxicol. $2002 ; 14: 417-23$.

2. Baselt R.C. Gamma-hydroxybutyrate. Disposition of toxic drugs and chemicals in man. 6th edition. Foster City, CA, Chemical Toxicology Institute, 2002 : 472-5.

3. Fieler E.L., Coleman D.E., Baselt R.C. Gamma-hydroxybutyrate concentrations in pre- and postmortem blood and urine. Clin. Chem. $1998 ; 44: 692$.

4. Stephens B.G., Coleman D.E., Baselt R.C. In vitro stability of endogenous gamma-hydroxybutyrate in postmortem blood. J. Forensic Sci. $1999 ; 44: 231$.

5. Elliott $S$. The presence of gamma-hydroxybutyric acid (GHB) in postmortem biological fluids. J. Anal. Toxicol. $2001 ; 25: 152$.

6. Kintz P. Difficultés analytiques et d'interprétation dans les expertises toxicologiques liées à la présence de GHB. J. Med. Legal Droit Med. 2002 ; 45 : 293-7.

7. Villain M., Cirimele V., Ludes B. , Kintz P. Ultra-rapid procedure to test for gamma-hydroxybutyric acid in blood and urine by gas chromatography-mass spectrometry. J. Chromatogr. B $2003 ; 792$ : 83-7.
8. Marinetti L.J., Isenschmid D.S., Hepler B.R., Schmidt C.J., Somerset S., Kanluen S. Two gamma-hydroxybutyric acid (GHB) facilities. Proceedings of the Annual Meeting of the American Academy of Forensic Science, Reno, 2000, résumé K16.

9. Kalasinsky K.S., Dixon M.M., Schmunk G.A., Kish S.J. Blood, brain, and hair GHB concentrations following fatal ingestion. J. Forensic Sci. 2001 ; 46 : 728-730.

10.Win B.H., Baselt R.C. Apparent suicide with Renewtrient. Clin. Tox. $2000 ; 38: 809$.

11. Elliott S.P. Further evidence for the presence of GHB in postmortem biological fluid: implications for the interpretation of findings. J. Anal. Toxicol. $2004 ; 28$ : 20-6.

12. Ferrara S.D., Tedeschi L., Frison G., Rossi A. Fatality due to gamma-hydroxybutyric acid (GHB) and heroin intoxication. J. Forensic Sci. 1995 ; 40 : 501-4.

13. Elliott S., Lowe P., Symonds A. The possible influence of micro-organisms and putrefaction in the production of GHB in post-mortem biological fluid. Forensic Sci. Int. $2004 ; 139: 183-90$. 